\title{
Compliance comparison of different bracing concepts
}

\author{
Andreas Selle*, Jens Seifert, Carl Gustav Carus \\ From 7th International Conference on Conservative Management of Spinal Deformities \\ Montreal, Canada. 20-22 May 2010
}

\section{Introduction}

Success in AIS-Bracing depends completely on patients' compliance. Compliance is an irreplaceable condition of IAS-Bracing. The investigation tries to find out differences in compliance between different bracing-methods and it compares especially Full-Time- and Night-Time-Bracing.

\section{Material and methods}

200 AIS patients (70 traditional Full-Time-Braces, 96 double treatments with traditional Day-Brace plus additional Night-Time-Brace and 34 patients with isolated Night-Time-Braces) have been included in a comprehensive, anonymous compliance survey. We distinguished daytime- and nighttime-compliance and various kinds of activity. We also asked patients and parents for their preferences concerning Early Night-Time-Bracing (ENTB).

\section{Results}

Among the users of traditional Full-Time-Braces we found an noncompliance rate of more than $40 \%$ during daytime. All of the braces were worn much more regularly during the night. Noncompliance rates for night use ranged from 15.3\% (traditional Full-Time-Braces) to only $8 \%$ for isolated Night-Time-Braces. $86.6 \%$ of the patients indicated that their quality of life (QOL) was most affected during daytime use and only $7.2 \%$ during night use. $98 \%$ of patients and parents preferred ENTB and were against waiting for verification of progression, because the avoidance of Full-Time-Treatment had highest priority for them. But facts are completely reverse: Only 13\% of AIS brace-patients get an EarlyNight-Time-Treatment. The majority of patients are still getting treatment at more than $25^{\circ} \mathrm{Cobb}$ and ad hoc 23 hours.

\footnotetext{
Orthopaedie- und Rehatechnik Dresden GmbH, Dresden, Germany
}

Full list of author information is available at the end of the article

\section{Discussion}

All the results of our compliance research show that physical and psychological stress of AIS Full-Time-Bracing is much higher than with Night-Time-Bracing, and the compliance rates are much lower during the day. Patients and parents want progression to be stopped as soon as possible to be, in particular, sure to avoid FullTime-Bracing. On the other hand, success of bracing is $100 \%$ dependent on patients' compliance. All of these facts underline the need for a treatment concept which can avoid AIS progression effectively while at the same time causing a minimal effect on the QOL. The most careful way of bracing is ENTB of moderate curvatures. In the range of $16-25^{\circ} \mathrm{Cobb}$ it is possible to stop progression and avoid Full-Time-Bracing with a success rate of $85 \%$ [1] by only using Night-Time-Braces with moderate correction forces, whereon patients respond with best acceptance.

\section{Conclusion}

By far the best compliance of AIS brace-treatment we found among patients who wear Night-Time-Braces at a point of moderate curvatures and enjoy daytime life without a brace. Their curves can be stopped with minimal effect on their QOL. Accordingly, in the future ENTB should become an integral part of AIS-therapy in between only physiotherapy and Full-Time-Bracing.

\section{Published: 10 September 2010}

\section{Reference}

1. Andreas Selle, Jens Seifert, Carl Gustav Carus: Early night-time-bracing - an alternative in AIS management. Scoliosis 2010, 5(Suppl 1):O57.

doi:10.1186/1748-7161-5-S1-059

Cite this article as: Selle et al:: Compliance comparison of different bracing concepts. Scoliosis 2010 5(Suppl 1):O59. 